\section{Experiência de cárie dentária em crianças de escolas públicas e privadas de um município com água fluoretada}

\author{
Dental caries experience in children \\ at public and private schools from \\ a city with fluoridated water
}

\author{
Rosana Helena Schlittler Hoffmann 1 \\ Silvia Cypriano 2 \\ Maria da Luz Rosário de Sousa 1 \\ Ronaldo S. Wada 1
}

\section{Introdução}

\footnotetext{
1 Departamento de Odontologia Social da Faculdade de Odontologia de Piracicaba, Universidade Estadual de Campinas, Piracicaba, Brasil.

2 Programa de Pós-Graduação em Odontologia, Faculdade de Odontologia de Piracicaba Universidade Estadual de Campinas,Piracicaba, Brasil.

Correspondência Maria da Luz Rosário de Sousa Departamento de Odontologia Social, Faculdade de Odontologia de Piracicaba, Universidade Estadual de Campinas. Av. Limeira 901, Piracicaba, SP 13414-900, Brasil. luzsousa@fop.unicamp.br
}

\begin{abstract}
The aim of this study was to verify the relationship between type of school as a measure of socioeconomic conditions and caries prevalence among preschoolers and schoolchildren in Rio Claro, São Paulo State, Brazil, a city with fluoridated water supply. The data were secondary, from a sample of 888 children 5 to 12 years old enrolled in private and public schools. Caries was measured by the dmft and DMFT indices as well as the Care index. Qui-square and MannWhitney tests were utilized with 5\% significance. In 5-year-old children, mean dmft was 2.50, and $42.20 \%$ were caries-free. At age 12, mean DMFT was 2.70 and $28.90 \%$ were cariesfree. Caries prevalence rates in public schoolchildren as compared to private were $74.50 \%$ and $61.20 \%$, respectively $(p<0.0001)$, and the dmft and DMFT scores were the highest in public schoolchildren $(p<0.05)$. The Care Index was higher in private schoolchildren (71.20\%) as compared to public (52.80\%). Highest caries rates were found among public schoolchildren, so the variable type of school proved sensitive for discriminating different oral health conditions; however limitations need to be recognized, suggesting that other variables should be assessed.
\end{abstract}

Oral Health; Social Class; Dental Caries; DMFT Index
Tem sido relatado em diferentes estudos epidemiológicos um declínio na prevalência de cárie dental em crianças de muitos países desenvolvidos 2,3,4,5,6,7. No Brasil, na década de 80, a prevalência de cárie em crianças de 12 anos encontrava-se entre as mais altas do mundo 8 , porém, alguns estudos realizados na última década revelaram uma significante redução na prevalência da cárie dentária 4,9,10,11,12 .

A maioria desses estudos verificou a prevalência de cárie em escolares do ensino público $4,10,13$, entretanto, também é relevante que sejam investigadas as condições de saúde bucal das crianças de escolas particulares, pois os levantamentos epidemiológicos realizados nas três últimas décadas já mostraram uma desigualdade de experiência de cárie entre as classes sociais 9,11,14,15,16,17,18,19. De acordo com Irigoyen et al. 20, o tipo de escola freqüentada é um indicador confiável de condição sócio-econômica em ambientes urbanos, pois crianças de famílias com altos salários geralmente freqüentam escolas particulares, sendo o oposto nas famílias com baixo nível sócio-econômico, as quais freqüentam principalmente o sistema público escolar.

Assim, o objetivo deste estudo foi verificar a relação entre o nível sócio-econômico, medido por meio da variável tipo de escola, e a prevalência de cárie em pré-escolares e escolares 
de um município com água fluoretada, localizado na Região Sudeste do Brasil.

\section{Métodos}

Os dados obtidos para este estudo são secundários, sendo a população de referência constituída de escolares de 5 a 12 anos do Município de Rio Claro, São Paulo, que provêm de um estudo transversal mais abrangente, envolvendo 133 municípios do Estado de São Paulo, estudo este que foi operacionalizado pela Secretaria de Estado da Saúde de São Paulo e Faculdade de Saúde Pública da Universidade de São Paulo (FSP/USP), em 1998 21. A amostra foi probabilística e o seu tamanho calculado conforme recomendação da FSP/USP 21, admitindose perda de elementos amostrais de $20,00 \%$ e erro de desenho igual a dois, que corresponde ao erro de estimação da amostra em função da técnica adotada. A amostra final foi de 888 crianças, constituída por alunos de escolas públicas e particulares, de ambos os sexos, permitindo inferência por tipo de escola no município para o grupo de 5 a 12 anos como um todo.

A pesquisa foi aprovada pelo Comitê de Ética em Pesquisa da FSP/USP (Processo COEP/ 62/98) e foram envolvidas as 24 Direções Regionais de Saúde do Estado de São Paulo. Um dos municípios sorteados para compor a amostra estadual foi Rio Claro, que se localiza na região sudeste desse Estado e dista $170 \mathrm{~km}$ da capital, tem uma população estimada de $174 \mathrm{mil}$ habitantes e aproximadamente $99,20 \%$ com acesso à água de abastecimento público fluoretada 22 .

Os exames clínicos foram realizados após a obtenção da autorização dos participantes valendo-se do termo de consentimento livre e esclarecido.

Os índices utilizados para coleta dos dados obedeceram aos critérios de diagnóstico recomendados pela Organização Mundial da Saúde (OMS) 1. As seguintes condições foram pesquisadas: cárie dentária (medida utilizando-se os índices ceod e CPOD), necessidade de tratamento, condição periodontal e a fluorose dentária. Entretanto, neste estudo, serão abordadas a experiência de cárie e a necessidade de tratamento.

A calibração foi realizada em 36 horas, divididas entre discussões teóricas e atividades práticas simulando as diferentes condições e situações que a equipe de 11 examinadores en- contraria durante a realização do trabalho prático. Para este treinamento, a técnica utilizada foi a do consenso e as discordâncias interexaminadores encontradas em relação à condição dental foi de $2,50 \%$, sendo considerada aceitável segundo a OMS. 23 A coleta de dados foi realizada nos meses de setembro a dezembro de 1998, e os dados foram digitados utilizando-se o software Epi-Info versão 5.01 e o Programa EPIBUCO para o processamento e análise dos dados 21 .

Os exames foram realizados sem iluminação artificial, utilizando-se sondas CPI ("ballpoint”) e espelhos bucais. Aproximadamente $10 \%$ da amostra foi reexaminada durante a fase de coleta dos dados, a fim de se verificar a discordância intra-examinadores. Este valor foi de $1,60 \%$ para a condição dental.

Foram também utilizados para a análise dos resultados, o Índice de Cuidados (Care Index) 24 , que mostra os cuidados restauradores a que a população alvo esteve exposta, por meio da relação de dentes obturados/CPO x 100. Como se tratava de dentição mista, utilizou-se a somatória de dentes decíduos e permanentes obturados no numerador e a soma ceo e CPO no denominador.

Considerou-se como prevalência de cárie as crianças que apresentaram ceod e CPOD maior que zero, assim, livres de cárie foram as que não apresentaram experiência de cárie na dentição decídua e permanente (ceod e CPOD =0).

Utilizou-se o teste qui-quadrado para se testar as diferenças entre a freqüência das variáveis nas escolas públicas e particulares no grupo de crianças de 5 a 12 anos, e o teste MannWhitney para verificar se houve diferenças entre os valores dos índices ceod e CPOD nos grupos estudados. Foi adotado o nível de significância estatística de $5 \%$.

\section{Resultados}

Examinaram-se 888 escolares de 5 a 12 anos, sendo $72,70 \%$ estudantes de escolas públicas variando de $70,40 \%$ a $77,50 \%$, e $27,30 \%$ de escolas particulares variando de $22,50 \%$ a $29,60 \%$ do Município de Rio Claro (Tabela 1).

Aos cinco anos o índice ceod foi de 2,48, aumentando proporcionalmente até a idade de oito anos, sendo que $42,20 \%$ não apresentaram experiência de cárie $(\operatorname{ceod}+\mathrm{CPOD}=0)$. Após essa idade, o índice ceod vai decrescendo até atingir 0,42 aos 12 anos. Paralelamente a isto, o 
índice CPOD aumentou proporcionalmente conforme a idade, até atingir a média de 2,71 aos 12 anos (Figura 1), sendo que $28,90 \%$ não apresentaram experiência de cárie.

A seguir, a experiência de cárie no grupo de crianças de 5 a 12 anos foi analisada segundo a variável estudada (tipo de escola). As crianças das escolas particulares apresentaram menores índices de cárie dentária (ceod e CPOD) e maior proporção de dentes obturados, medidos pelo Índice de Cuidados. O Índice de Cuidados também foi maior nestes escolares (Tabela 2). Entretanto, o ceod e CPOD, calculados

Tabela 1

Número de indivíduos examinados, segundo o tipo de escola e a faixa etária. Rio Claro, São Paulo, Brasil, 1998.

\begin{tabular}{crrrrrr}
\hline \multirow{2}{*}{ Idade (anos) } & \multicolumn{4}{c}{ Tipo de escola } & \multicolumn{2}{c}{ Total } \\
& \multicolumn{2}{c}{ Pública } & \multicolumn{2}{c}{ Particular } & & \\
& $n$ & $\%$ & $\mathrm{n}$ & $\%$ & $\mathrm{n}$ & $\%$ \\
\hline 5 & 75 & 73,5 & 27 & 26,5 & 102 & 100,0 \\
6 & 71 & 71,7 & 28 & 28,3 & 99 & 100,0 \\
7 & 77 & 74,8 & 26 & 25,2 & 103 & 100,0 \\
8 & 70 & 71,4 & 28 & 28,6 & 98 & 100,0 \\
9 & 79 & 77,5 & 23 & 22,5 & 102 & 100,0 \\
10 & 78 & 70,9 & 32 & 29,1 & 110 & 100,0 \\
11 & 101 & 72,7 & 38 & 27,3 & 139 & 100,0 \\
12 & 95 & 70,4 & 40 & 29,6 & 135 & 100,0 \\
Total & 646 & 72,7 & 242 & 27,3 & 888 & 100,0 \\
& & & & & &
\end{tabular}

excluindo as crianças livres de cárie, não apresentaram diferença estatística significativa frente ao nível socioeconômico, como também não diferiu quanto à porcentagem das crianças com ceod maior que três.

Foi observada maior porcentagem de crianças com CPOD maior que três no grupo de crianças de escolas públicas do que no de ensino particular $(p=0,005)$, conforme apresentado na Tabela 2 .

\section{Discussão}

Este trabalho visa a contribuir para uma discussão mais abrangente sobre a relação saúde bucal e nível sócio-econômico, tendo em vista a carência de trabalhos que utilizam a variável tipo de escola como indicador de nível sócioeconômico em saúde bucal. Contudo, os trabalhos de literatura apontam limitações na coleta de dados sócio-econômicos, principalmente quando a população em estudo se refere a escolares, pois muitas vezes torna-se necessário o envolvimento dos familiares para avaliações mais precisas, dificultando a operacionalização desse tipo de estudo. Assim, neste trabalho, apesar das limitações desta variável selecionada (tipo de escola) optou-se por estudála, corroborando com outros estudos 9,11,17,20.

Apesar de outras variáveis sócio-econômicas como a renda familiar, escolaridade do pai e número de residentes por cômodos 18 serem importantes para diferenciar os níveis sócio-

Figura 1

Índice ceod e CPOD (IC95\%) segundo a idade. Rio Claro, São Paulo, Brasil, 1998.

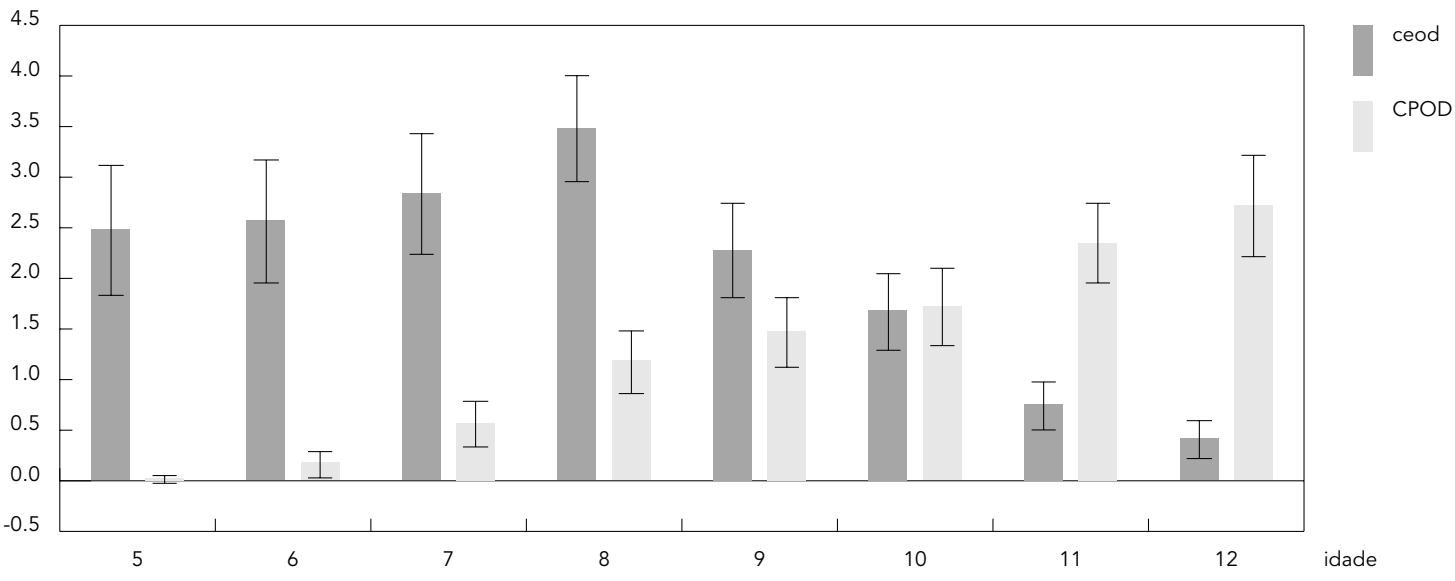


Experiência de cárie na dentição decídua e na permanente, segundo o tipo de escola. Rio Claro, São Paulo, Brasil, 1998.

\begin{tabular}{|c|c|c|c|}
\hline \multirow[t]{2}{*}{ Variáveis } & \multicolumn{2}{|c|}{ Tipo de escola } & \multirow[t]{2}{*}{ Valor de $p$} \\
\hline & Pública $(n=646)$ & Particular ( $n=242)$ & \\
\hline Ceod $(d p)^{\star}$ & $2,05 \mathrm{a}(2,66)$ & $1,62 b(2,38)$ & $0,0086^{\star *}$ \\
\hline Ceod dentre os doentes $(\mathrm{dp})^{\star}$ & 3,74 a $(2,85)$ & 3,74 a $(2,27)$ & $0,5926^{\star *}$ \\
\hline Crianças com ceod $=0(\%)$ & $45,05 a$ & $56,61 b$ & 0,0021 \\
\hline Crianças com ceod > $3(\%)$ & $24,00 a$ & $20,70 a$ & 0,2940 \\
\hline Índice de cuidados - dentição decídua (\%) & $52,00^{a}$ & $76,30 \mathrm{~b}$ & $<0,0001$ \\
\hline CPOD $(\mathrm{dp})^{\star}$ & 1,53 a $(2,23)$ & $1,00 \mathrm{~b}(1,63)$ & $0,0013^{\star \star}$ \\
\hline CPOD dentre os doentes $(d p)^{*}$ & 3,37 a $(2,18)$ & $2,93 a(227)$ & $0,1761^{\star *}$ \\
\hline Crianças com CPOD = $0(\%)$ & $54,64 a$ & $65,70 \mathrm{~b}$ & 0,0030 \\
\hline Crianças com CPOD > $3(\%)$ & $21,10 a$ & $2,90 b$ & 0,0051 \\
\hline Índice de cuidados - dentição permanente (\%) & $53,80 a$ & $63,00 b$ & 0,0105 \\
\hline Prevalência de cárie (ceod e CPOD > 0) & $74,50 a$ & $61,20 b$ & 0,0001 \\
\hline Crianças livres de cárie (ceod e CPOD = 0) (\%) & $25,50 a$ & $38,80 \mathrm{~b}$ & 0,0001 \\
\hline Índice de cuidados (\%) & $52,80 a$ & $71,20 b$ & $<0,0001$ \\
\hline
\end{tabular}

Números seguidos de letras diferentes na horizontal diferem entre si com significância de $5 \%$.

* Desvio padrão.

** Para testar as médias ceod e CPOD utilizou-se o Mann-Whitney test.

Os demais valores de $p$ foram calculados pelo qui-quadrado.

econômicos, o presente estudo, utilizando o tipo de escola, encontrou diferenças na experiência de cárie entre os grupos pesquisados em ambas as dentições.

O índice ceod aos cinco anos das crianças de Rio Claro $(2,48)$, foi próximo ao encontrado em Blumenau, Santa Catarina $(2,42)$, e ao se comparar os dados por tipo de escola, encontraram melhores condições nas crianças da rede particular de ensino, concordando com os achados deste estudo 11.

Os resultados deste estudo, entretanto, foram menores que os índices observados por Freire et al. 13, em municípios goianos, que apresentaram ceod de 4,93 aos cinco anos, denotando melhores condições de saúde bucal em Rio Claro. Já em Dom Aquino, Mato Grosso, este dado foi de 6,60 e nos escolares de 6 a 12 foi de 3,97 25, ambos também mais elevados do que os de Rio Claro, inclusive ao comparar-se com as crianças da rede pública.

Dados internacionais, por outro lado, apontam para um índice ceod baixo aos cinco anos, como por exemplo, na Grã Bretanha cujo ceod foi de 1,52 em 2001/2002, com variação de 0,75 a 2,47 26. Este quadro, porém, modifica-se quando o índice é calculado excluindo-se as crianças sem experiência de cárie $(\operatorname{ceod}=0)$, passando para uma média de 3,83 nos escolares da Grã-Bretanha, sendo esse valor próximo ao encontrado nas crianças de Rio Claro $(3,74)$, não variando em função do tipo de escola em Rio Claro.

Esses resultados sugerem que no grupo de crianças com experiência de cárie, considerando a dentição decídua, a severidade da doença é alta, mesmo em localidades economicamente mais desenvolvidas como a Grã-Bretanha 26 ou em estratos sociais mais favorecidos economicamente. Isso salienta a importância em se planejar estratégias adequadas para cada grupo da população, visando a reduzir a ocorrência e a severidade da doença também dentre os que são mais acometidos, sendo importante que outros índices, além do ceod, sejam analisados durante o planejamento das ações.

Considerando a dentição permanente, a prevalência de cárie no município foi considerada moderada $(2,71)$, atingindo as metas estabelecidas pela OMS para o ano 2000 na idade de 12 anos. Valores menores foram observados em Salvador, Bahia, no ano de 2001 9; Blumenau, em $1998(1,46)$ 11; na cidade de São Paulo em 1996 (2,06) 12; e em Araraquara, São Paulo, em $1995(2,60) 4$, entretanto, outras localidades do Brasil como Bauru, São Paulo 10 e Goiás 13, apresentaram índices mais elevados, sendo respectivamente, de 3,42 e 5,19; sendo maior também na Cidade do México que foi de 4,40 20. Na Grã Bretanha, 26 o CPOD aos 12 anos foi de 0,89 , entretanto encontrou-se uma média de 2,35 ao se excluir as crianças que não apre- 
sentaram experiência de cárie na dentição permanente, sendo que esse resultado ficou mais próximo ao valor encontrado nos escolares com experiência de cárie do ensino privado de Rio Claro $(2,93)$.

Deve-se, entretanto, considerar que as crianças de escolas públicas com experiência de cárie apresentaram um índice ainda mais elevado $(3,37)$, denotando maior severidade da doença nessas crianças, sendo importante que outros índices também sejam utilizados durante o planejamento, pois seria de fundamental importância que se reduzisse não apenas a média de dentes com cárie, mas também neste grupo com experiência de cárie, bem como se aumentasse a proporção de indivíduos livres de cárie. Esse enfoque encontra justificativa teórica no fenômeno da polarização, segundo o qual cerca de $25,00 \%$ dos indivíduos concentram aproximadamente $75,00 \%$ dos dentes com prevalência de cárie 12 .

Considerando os escolares de 5 a 12 anos, os valores de prevalência de cárie deste estudo foram abaixo dos encontrados nos escolares tanto do ensino público $(67,50 \%)$ como do privado $(54,50 \%)$ da Cidade do México 20 , sendo as diferenças estatisticamente significantes em ambos os estudos.

Enfatiza-se que tanto na dentição decídua quanto na permanente, considerando o grupo de crianças com maior experiência de cárie (ceod ou CPOD maior que três), não houve diferença na média destes índices entre os escolares do ensino público e particular, denotando que a severidade da cárie foi semelhante em ambos os grupos. Fato este importante na ocasião em que forem traçadas estratégias de planejamento.

Ao se discriminar o tipo de escola, foram verificadas diferenças estatisticamente significativas do CPOD, sendo maior nos estudantes do ensino público, discordando dos achados de Cangussu et al. 9 e Narvai et al. 12, apesar da amostra desses estudos terem sido bem maiores do que a de Rio Claro. Sob o aspecto de componentes do índice, não houve discordância entre os estudos, predominando o componente obturado em estudantes de estabelecimentos privados e o componente cariado nos estudantes dos estabelecimentos públicos, reforçando assim a diferença de acesso a cuidados em saúde bucal nesses estudantes.

Constatou-se um Índice de Cuidados elevado na dentição decídua, sendo maior na rede privada $(76,30 \%)$ do que na pública $(52,00 \%)$. Ambos os resultados foram mais elevados do que os dados observados na Grã Bretanha $(13,20 \%) 26$ e no ensino público de Blumenau,
Santa Catarina $(16,80 \%) 11$, em escolares aos cinco anos de idade.

Na dentição permanente, o Índice de Cuidados nas crianças da rede particular de ensino de Rio Claro, foi de $63,00 \%$ e 53,80\% na pública, sendo ambos os valores abaixo dos relatados por Cangussu et al. 9 em Salvador $(71,40 \%$ e $57,70 \%$, respectivamente), mas acima do resultado encontrado nos escolares de 6 a 12 anos em Dom Aquino, por Morais et al. 25, cujo valor foi de $11,60 \%$. Nos escolares de 7 a 12 anos da rede pública de Araraquara 4, o Índice de Cuidados mostrou-se mais elevado, variando de $70,90 \%$ a $90,80 \%$.

Assim, os dados deste estudo de Rio Claro, São Paulo, confirmaram que o Índice de Cuidados é importante não só para o planejamento de estratégias, como também sendo um importante indicador dos serviços de saúde, pois refletem os cuidados restauradores para os indivíduos com necessidade de tratamento 26 .

Considerando o tipo de ensino, o Índice de Cuidados também foi sensível para discriminar os diferentes grupos, refletindo que as crianças com menor acesso aos cuidados em saúde bucal pertenciam a setores economicamente menos favorecidos.

\section{Conclusões}

As diferenças encontradas neste estudo são consistentes com achados das literaturas nacional e internacional, salientando que com base nos resultados para o tipo de escola, a experiência de cárie e necessidade de tratamento foram maiores nas crianças que freqüentavam escolas públicas, ressaltando as limitações deste presente estudo quanto à utilização da variável tipo de escola como indicador de nível sócio-econômico.

Adquirir um conhecimento detalhado da distribuição da cárie e seus determinantes biopsicossociais é o passo inicial e indispensável para o planejamento de programas em saúde bucal. Assim, incluir variáveis relacionadas ao nível sócio-econômico torna-se fundamental, bem como a utilização de outros índices e indicadores que não apenas o ceod e o CPOD. 


\section{Resumo}

O objetivo deste estudo foi verificar a relação entre tipo de escola, como medida de condição sócio-econômica e a prevalência de cárie em pré-escolares e escolares de Rio Claro, São Paulo, com água fluoretada. Os dados obtidos são secundários e a amostra foi de 888 escolares de 5 a 12 anos dos ensinos público e particular. A experiência de cárie foi medida por meio dos índices ceod e $C P O D{ }^{1}$, além do Índice de Cuidados. Empregou-se os testes qui-quadrado e Mann-Whitney com significância de 5\%. Aos cinco anos, o ceod foi de 2,50 e 42,20\% não apresentaram experiência de cárie. Aos 12 anos, o CPOD foi de 2,70 e 28,90\% estavam livres de cárie. A prevalência de cárie nas crianças de escolas públicas foi maior do que nas particulares,sendo respectivamente de 74,50 e 61,20\% ( $p<0,0001$ ), as sim como os indices ceod e CPOD $(p<0,05)$. O Índice de Cuidados foi maior nas crianças do ensino particular $(71,20 \%)$ do que nas do ensino público (52,80\%). Encontrou-se uma maior experiência de cárie nos escolares do ensino público e assim a variável tipo de escola foi sensível para discriminar diferentes condições de saúde bucal, sugerindo-se que outras variáveis também sejam avaliadas.

Saúde Bucal; Classe Social; Cárie Dentária; Índice CPOD.

\section{Colaboradores}

R. H. S. Hoffmann contribuiu com a idéia original do tema, revisão bibliográfica, introdução, discussão dos resultados e redação final do artigo. S. Cypriano participou da realização da metodologia, análise e discussão dos resultados. M. L. R. Sousa colaborou na coordenação do trabalho, discussão dos resultados e elaboração das conclusões. R. S. Wada participou do planejamento da pesquisa, análise dos resultados e elaboração das conclusões.

\section{Agradecimentos}

Os autores agradecem a todos que autorizaram que os exames fossem realizados, aos coordenadores de saúde bucal dos municípios, cirurgiões-dentistas e auxiliares que contribuíram para que esta pesquisa fosse realizada. Em especial à cirurgiã dentista Roberta Dalcico, pelas suas importantes contribuições. À bibliotecária Marilene Girello pelo auxílio nas correções das referências bibliográficas.

\section{Referências}

1. World Health Organization. Oral health surveys, basic methods. Geneva: World Health Organization; 1997.

2. Bönecker M, Cleaton-Jones P. Trends in dental caries in Latin American and Caribbean 5-6 and 11-13-year-old children: a systematic review. Community Dent Oral Epidemiol 2003; 31:152-7.

3. Pitts NB, Evans DJ, Nugent ZJ, Pine CM. The dental caries experience of 12-year-old children in England and Wales. Surveys coordinated by the British Association for the Study of Community Dentistry in 2000/2001. Community Dent Health 2002; 19:46-53.

4. Dini EL, Foschini ALR, Brandão IMG, Silva SRC. Changes in caries prevalence in 7-12 year-old children from Araraquara, SP, Brasil: 1989-1995. Cad Saúde Pública 1999; 15:617-21.

5. Marthaler TM, O'Mullane DM, Vrbic V. The prevalence of dental caries in Europe 1990-1995. Caries Res 1996; 30:237-55.

6. Petersson HG, Bratthall D. The caries decline: a review of reviews. Eur J Oral Sci 1996; 104:436-43.

7. Burt B. Trends in caries prevalence in North American children. Int Dent J 1994; 44:403-13.

8. Ministério da Saúde. Levantamento epidemiológico em saúde bucal: Brasil, zona urbana, 1986. Brasília: Divisão Nacional de Saúde Bucal, Centro de Documentação, Ministério da Saúde; 1988. 
9. Cangussu MCT, Castellanos RA, Pinheiro MF, Albuquerque SR, Pinho C. Cárie dentária em escolares de 12 e 15 anos de escolas públicas e privadas de Salvador, Bahia, Brasil, em 2001. Pesqui Odontol Bras 2002; 16:379-84.

10. Bastos RS, Bijella VT, Bastos JRM, Buzalaf MAR. Declínio da cárie dentária e incremento no percentual de escolares, de 12 anos de idade, livres da doença, em Bauru, SP, entre 1976-1995. Revista da Faculdade de Odontologia de Bauru 2002; 10:75-80.

11. Traebert JL, Peres MA, Galesso ER, Zabot NE, Marcenes W. Prevalência e severidade da cárie dentária em escolares de seis a doze anos de idade. Rev Saúde Pública 2001; 35:283-8.

12. Narvai PC, Castellanos RA, Frazão P. Prevalência de cárie em dentes permanentes de escolares no Município de São Paulo, SP, 1970-1996. Rev Saúde Pública 2000; 34:196-200.

13. Freire MCM, Pereira MF, Batista SMO, Borges MRS, Barbosa MI, Rosa AGF. Prevalência de cárie e necessidades de tratamento em escolares de 6 a 12 anos da rede pública de ensino. Rev Saúde Pública 1999; 33:385-90.

14. Tinanoff N, Kanellis MJ, Vargas CM. Current understanding of the epidemiology, mechanisms and prevention of dental caries in preschool children. Pediatr Dent 2002; 24:543-51.

15. Baldani MS, Narvai PC, Antunes JLF. Cárie dentária e condições sócio-econômicas no Estado do Paraná, Brasil, 1996. Cad Saúde Pública 2002; 18:755-63.

16. Antunes JLF, Frazão P, Narvai PC, Bispo CM, Pegoretti $\mathrm{T}$. Spatial analysis to identify differentials in dental needs by área-based measures. Community Dent Oral Epidemiol 2002; 30:133-42.

17. Maltz M, Silva BB. Relação entre cárie, gengivite, fluorose e nível sócio-econômico em escolares. Rev Saúde Pública 2001; 35:170-6.
18. Peres KGA, Bastos JRM, Latorre MRDO. Severidade de cárie em crianças e relação com aspectos sociais e comportamentais. Rev Saúde Pública 2000; 34:402-8.

19. Al-Mohammedi SM, Rugg-Gunn AJ, Butler TJ. Caries prevalence in boys aged 2, 4 and 6 years according to socio-economic status in Riyadh, Saudi Arabia. Community Dent Oral Epidemiol 1997; 25:184-6.

20. Irigoyen ME, Maupome G, Mejfa AM. Caries experience and treatment needs in a 6- to 12-year-old urban population in relation to socio-economic status. Community Dent Health 1999; 16:245-9.

21. Narvai PC, Castellanos RA. Levantamento das condições de saúde bucal - Estado de São Paulo, 1998. São Paulo: Núcleo de Estudos e Pesquisas de Sistemas de Saúde, Secretaria de Estado da Saúde de São Paulo; 1999.

22. Fundação Sistema Estadual de Análise de Dados. Perfil Municipal de Rio Claro. http://www.seade. gov.br/cji-bin/perfil/request.ksh?PM20379 (acessado em 30/Abr/2003).

23. World Health Organization. Basic methods. Geneva: World Health Organization; 1987.

24. Walsh J. International patterns of oral health care - the example of New Zealand. N Z Dent J 1970; 66:143-52.

25. Morais ND, Lenza MA, Freire MCM. Prevalência de cárie em escolares de 6 a 12 anos da rede pública de ensino do Município de Dom Aquino MT. Revista Brasileira de Odontologia Coletiva 2000; 1:45-9.

26. Pitts NB, Boyles J, Nugent ZJ, Thomas N, Pine CM. The dental caries experience of 5-year-old children in England and Wales. Surveys coordinated by the British Association for the Study of Community Dentistry in 2001/2002. Community Dent Health 2003; 20:45-54.

Recebido em 02/Jun/2003

Versão final reapresentada em 29/Ago/2003

Aprovado em 15/Set/2003 